\title{
An Analysis on the Emotion in the Field of Translator's Subjectivity
}

\author{
Wei Yuehong ${ }^{1, a}$ \\ Department of English, North China Electric Power University, Baoding, 071003, China \\ wyh231@163.com
}

Keywords: Emotional factors, Translation, Translator's Subjectivity, Multimodal Corpus Approach

\begin{abstract}
The translator's subjectivity has attracted more and more attention of scholars in the field of translation studies. The study of translator's subjectivity has appeared the trend of diversification since researchers have transferred their studies on translation from the perspective of the pure linguistic level to the study of the translator's own factors. The study of translator's subjectivity from the perspective of psychology and cognition has become the focus of attention of scholars, but the study of emotions in the translator's subjectivity is still in its infancy. This study aims to study the emotional factors in the translator's subjectivity from the perspective of psychology and cognitive science, and a multimodal corpus approach is analyzed to construct a model of translator's emotional change. The objects of this study include two parts: translation and interpretation. A model of translator's emotional change which includes words, thinking, and appearance will be discussed and it can be constructed by using the approach of a multimodal corpus. And a case study will be conducted to explore how to effectively control the training mode of emotion in the process of translation based on the analysis results. This study will provide valuable references for the study of translation teaching, training of translators, Machine Translation, and so on.
\end{abstract}

\section{Introduction}

The translator's subjectivity is constantly awakening with the development of translation studies in different times, but the translator's subjectivity is shown as different translator styles for it is influenced by various factors such as the background of the times, the original text, ideology, mainstream poetics, cultural differences, and so on. Moreover, some scholars have pointed out that the translator's subjectivity is not only influenced by the above factors, but also has subjective initiative. Since the 1990s, researchers have carried on thorough discussion on translator's subjectivity from perspectives of Hermeneutics, culture, feminism, deconstruction, and so on. Some scholars focus on the in-depth excavation of the translator's subjectivity(Cha Mingjian, 2003[1]; Li Ying, 2006[2]); some scholars have discussed the translator's subjectivity only from a single point of view (Zhang Shunsheng,2008[3];Liao Jing,2005[4]; Xiaowei Zheng,2011[5];Shu Huijuan,2012[6]); some scholars analyze the translator's subjectivity from the perspective of pluralism (Zhong Weihe, Zhou Jing,2006[7];Liu Junping,2008[8]; Zhao Zhihua,2011[9]). Mu Lei and Shi Yi (2003) [10] discussed the "discovery" and research of translator subjectivity from the perspective of translators' studies, in which they mentioned two articles, on is Emotional Transplantation in Literary Translation that is written by Zhang Cheng, and the other is Translation Is AKind of Encounter, which is written by Wang Binbin, emphasizing the importance of emotion of translator's subjectivity in translation. Moreover, they further mentioned that Shen Dan has pointed out through comparison of several translated texts in her article, On the Translator's Objectivity in the Translation of Novels, that the translator's objectivity may be unconsciously influenced by the translator's "practical interest", emotional and psychological factors, cognitive frame, and expectation horizon. In the study of translator's subjectivity, scholars have put forward that subjectivity is influenced by the translator's psychological factors like the translator's attitude and the translator's emotions. However, there is little research in this area. Scholar Zhao Zhihua (2011) [9] proposes that "survival mentality" should be considered as motivation of studies of translator's subjectivity, and he also expounds the concrete function of the passivity of subjectivity in the translator's behavior. These subjective factors can influence the translation process and translation results, therefore, translation process can not merely be seen as a simple language 
conversion process, but a subjective and objective complex. Consequently, in the basic qualities that the translator should have, in addition to having a good ability of bilingual translation, a good psychological quality is the basic requirement of the translator.

\section{Theoretical Basis}

The translator's subjectivity is the subjective initiative performed by the translator, who is the subject of translation, in translation activities in order to achieve the goal of translation under the premise of respecting the translation object. And its basic characteristics are the cultural consciousness, the cultural character, and the aesthetic creativity of the culture of the subject of translation. Recognizing and emphasizing the translator's subjectivity does not mean that the translator can be arbitrary but mean to strengthen the translator's self-consciousness and subject consciousness, so that the translator can better play his or her creativity. To play the translator's subjectivity, the translator must understand his or her inner structure and have the necessary qualities of a translator.

As the subject of translation, the translator has a complicated system structure, which is generally constructed of need-culture-psychological structure. The need structure includes the translator's own and social needs, that is, the translator should make translational selection before translation since need is the original motivation of subjective activities. But only need structure without proper cultural structure, the translator can do nothing but lament in translation practice since the cultural structure is the basis for the realization of the need structure. A qualified translator should at least have the following cultural qualities: solid language skills, rich cultural knowledge and be familiar with translation theory and skills. Finally, as the subjectivity of translation, the translator is not only driven and controlled by the rational consciousness, but also affected by emotional will system of the psychological level, such as the influence of factors like traditional and emotional will.

Researches have shown that affective factors play an important role in language learning process. Similarly, in the process of translation, namely, the stage of language use, the translator's emotions will have an impact on the translation process. The question of how to make the translator's emotions exert a positive impact on the subjectivity of the translators a cross disciplinary issue in psychology, linguistics, cognition and translation studies. At present, there are scholars analyzing the intervention of translator's emotions in the process of poetry translation in translation studies; some scholars study the emotional resonance between the translator and the author; some scholars analyze the translator's emotional involvement from the perspective of text type. Among these scholars, Qian Chunhua (2012) [11]constructed the translator's ecological system in her article, An Empirical Study on the Elements of Translation Ecological System based on the Translator's Cognition, in which she specially mentioned the translator's emotional factors in the category of the translator's factors. In the field of interpretation, community interpretation will gradually become a new hot spot in the study of interpreting for a long time in the future. These researches are almost descriptive as they makes objective description and system analysis of such issues as social communication activities based on interpretation services under special situation, especially the influence of interpreting activities on social activities, changes of interpretation nature and function in communicative activities, the interpreter's status, identity, role, as well as evaluation of quality and effectiveness of interpretation by drawing lessons from the theories and concepts of sociology, anthropology, communication and other related disciplines, and by means of quantitative analysis, case analysis as well as text interpretation. Some scholars point out that the interpreting studies should not just stay on the surface layer of language conversion, and that more efforts should be made to investigate and analyze the internal mechanism of interpretation. Moreover, empirical observation and analysis should be made on these issues such as understanding mechanism of interpretation thinking, psychological prediction mechanism of interpretation, the development of interpretation of the mental lexicon and extraction mode, working mode of interpreting memory, and information Units of Interpretation by referring to the concepts of psychology, psycholinguistics, cognitive psychology, communication and other related disciplines. In the 
process of studying these mechanisms, the main role of the translator is particularly important, and the affective factors of the interpreter should be regarded as important research content. For example, issues such as manifestations of changes of emotion, impact of emotional changes, and effective way of emotional control should be analyzed. However, the research on the translator's subjectivity of has been neglected. This study focuses on the translator's emotions, respectively studying the emotional state and changes of translators and interpreters in the process of translation and interpretation from perspectives of psychology and cognition. Moreover, a positive emotional model will be constructed, which provides translation studies with a research entrance from outside and inside, promotes the study of translation process and improves the teaching effect of translation.

\section{Research Design}

The main content of this research is emotional factors in the translator's subjectivity, and the changing rules of the translator's emotional factors will be analyzed from perspectives of psychology and cognitive science. Additionally, an emotional change model of translator will be constructed by means of multimodal corpus, and influence of emotion on translation effects will be analyzed by the whole principle of language, thinking, and appearance.

1) Research on translator's emotion: this research introduces the theory of psychology - theory of social culture, into the study of emotional factors in the translator's subjectivity. Guided by this theory, trends in emotional change will be analyzed by such means as questionnaires, interviews, observation, and human-computer interaction, etc.; the influence degree of emotional change on translation effects will be analyzed through investigation of translator and translator service object, and an association model of positive emotion and translation effects will be constructed. Based on the above two parts of researches, the author will further explore how to effectively control the training mode of emotion through case study.

2) Research on the emotion of interpreter: an emotional change model of translator, which includes language, thinking, and appearance, will be constructed by means of multimodal corpus. According to the researcher's classification, emotion includes: anxiety, exhaustion, lethargy, sickness, relaxation, health, interest and spirit. In the process of interpretation, these emotional factors of anxiety, exhaustion, lethargy, sickness, relaxation, health, interest and spirit will have great influence on interpretation. This research will make tracking service on the above emotional manifestations, and collect emotional changes characteristic corpus of subjects by means of questionnaire, interview, observation, real time video recording and analysis of the image data in the field of interpretation. And at the same time, the author will make tracking service on the corresponding interpretation effects, and construct an association mechanism of interpreter's emotion and interpretation effects. The data model will provide a reference for the translator's emotional control process through the analysis of correlation.

First of all, research on the practice of translation will be carried out with MTI translation and interpreting students as the research objects. And a questionnaire and interview survey on translator's emotional involvement will be compiled, which mainly focus on the three stages of before translation, in translation, and after translation. This research will be able to regulate MTI translation practice teaching activities and play a very good supervisory role to the teaching effect.

Then, the author will research on the influence degree of translator's emotional factors on translator's subjective initiative by using the research methods of questionnaire, interview and video recording with practitioners of professional translators and interpreters as objects of investigation. Generally speaking, translators or interpreters may ignore the influence of their subjective initiative on translation; therefore, arousing the translator's sense of self emotion mobilization is a major breakthrough in the study of translation subjectivity.

Finally, investigation of evaluators of translation works, which include translation employers, readers, conference audience, will be conducted to examine the above persons' affective feedback to translation or interpretation process and evaluation of translation effects. And comparative analysis between this data and the translator's emotional change process data will be made to get the results of the correlation analysis of the two. 


\section{Analysis of Research Results}

The purpose of this research is to construct an emotional change model of translators, and study the law of translators' emotional change according to the model. The emotional factors of translators in translation process are the subjects of this research, and the influence of affective factors on translators in translation process will be analyzed. Additionally, by constructing multi modal emotion description corpus, this research further discusses how to create and utilize positive affective effects in the social communication environment, which will provide a new perspective for the study of translator's subjectivity, and reference for translator training, as well as translation teaching and research. The emotional content of the study is shown in Fig. 1:

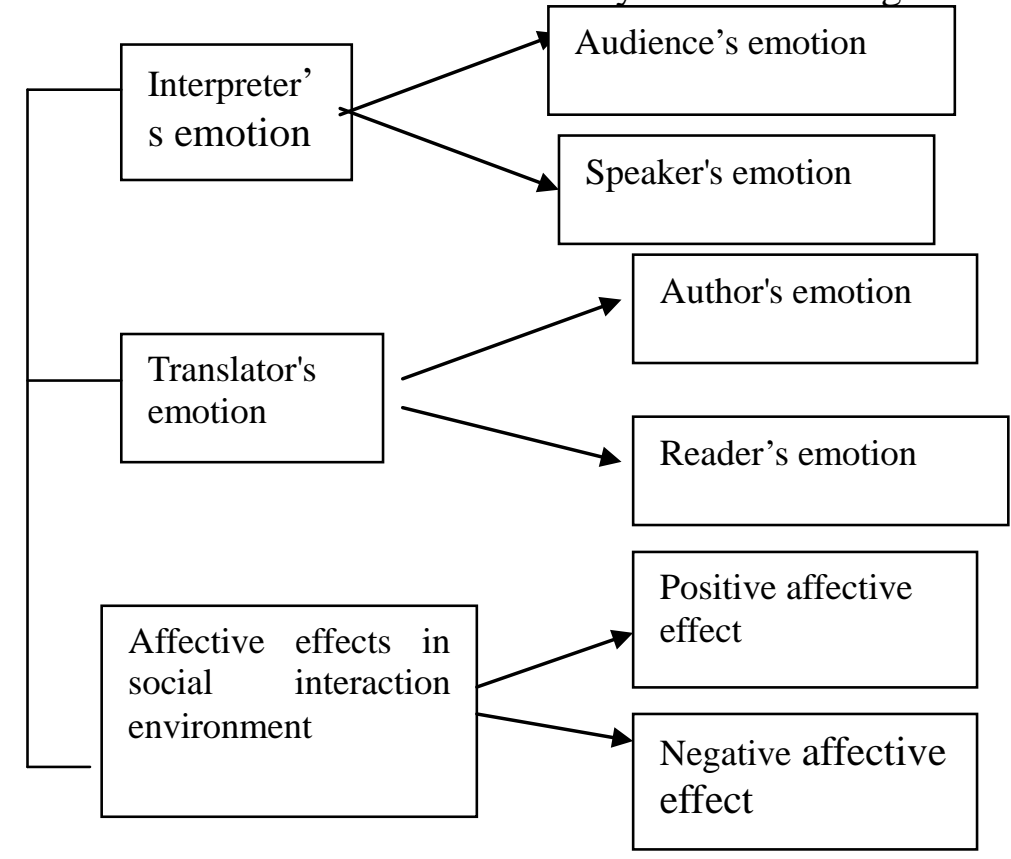

Fig.1Emotional Factors in Translation

The following hypotheses are tested by the research results: emotion may exert influence in translation process on such aspects as lexical choices, discourse analysis, logical thinking expression, identification of the author and speaker, deep thinking on the work and presentation materials, and so on. This research will prove the above hypotheses, put forward the internal mechanism of the translator's subjectivity by combining with the research results, and further explain the psychological and cognitive mechanism of the translator's subjectivity from perspective of translator's emotion.

\section{Conclusion}

Currently, the translation industry has entered a period of vigorous development, and at the same time, the translation major, as an emerging discipline, has been vigorously developed in many high schools. However, the high-end talent of the translation industry is still a gap, especially in the simultaneous interpretation industry, and it is not difficult to find the reason that this industry not only requires the translator to have a higher ability to use language, but also has higher requirements on the translator's own qualities. As for translators' own qualities, emotional factor of the translator is a major aspect; therefore, there should be a systematic emotional model as a guide for the training of senior interpreter and translator, which has been ignored by trade public Figures. Moreover, constructing a multimodal corpus is beneficial for translators or interpreters since it can help them see the discourse analysis of the original interpreter. This method is superior to the traditional experience teaching system, and will have an important guiding significance for the development of MTI teaching and invention of Machine Translation software. 


\section{Acknowledgment}

This research was supported by "Fundamental Research Funds for the Central Universities (Grant\# 2016MS135) and the Excellent Youth Teacher Supporting Project.

\section{References}

[1]. Mingjian Cha, Yu Tian. Discussion on the Translator's Subjectivity[J]. Chinese Translators Journal, 2003(1):22.

[2]. Ying Li. Discussion on the Inner Structure of Translator's Subjectivity[J]. Journal of Southwest University, 2006(11).

[3]. Shunsheng Zhang . The Bottom Line of the Translator's Subjectivity [J] . Shanghai Journal of Translators, 2008 ( 2 ): 63- 66 .

[4]. Jing Liao. Study on the Identity of Translator[J]. Chinese Translators Journal, 2005(3).

[5]. Xiaowei Zheng. On the Translator's Subjectivity in Translation Teaching in the Perspective of Cognitive Linguistics[J], Foreign Language and Literature, 2011(1):97-102.

[6]. Huijuan Su. Dynamic Projection and Translator's Subjectivity[J]. Shanghai Journal of Translators, 2012(3):13-15.

[7]. WeiheZhong, Jing Zhou. The Extreme Limit and the Bottom line-Analysis on the Translator's Subjectivity and the Duties of Translators [J]. Foreign Languages and Their Teaching, 2006(7):42-46.

[8]. Junping Liu. The Four Dimensions and Features of the Translator's Subjectivity from the Aspect of interdisciplinary [J]. Foreign Languages and Their Teaching, 2008(8):53.

[9]. Zhihua Zhao. 2009. Translator's Subjectivity from the Perspective of Habitus [D]. Jinhua: Zhejiang Normal University.

[10]. Lei Mu, Yi Shi. Study and “Discovery” of Translator's Subjectivity[J]. Chinese Translators Journal, 2003(1).

[11]. Chunhua Qian. The Experimental Research on the factors in Translation Ecological System Based on the cognition of Translators[J]. Foreign Language Education,2012(2). 